九州大学学術情報リポジトリ

Kyushu University Institutional Repository

\title{
COSISTENCY OF THE CORRELATION DIMENSION ESTIMATOR FROM A CHAOTIC SYSTEM
}

Kawaguchi, Atsushi

Graduate School of Mathematics, Kyushu University

https://doi.org/10.5109/13518

出版情報: Bulletin of informatics and cybernetics. 35 (1/2), pp.27-34, 2003-12. Research Association of Statistical Sciences

バージョン :

権利関係 : 


\title{
CONSISTENCY OF THE CORRELATION DIMENSION ESTIMATOR FROM A CHAOTIC SYSTEM
}

\author{
By
}

\section{Atsushi KaWAGUCHI*}

\begin{abstract}
In this paper, we prove the consistency of the correlation dimension estimator proposed by Kawaguchi(2002) for a deterministic chaotic system.
\end{abstract}

Key Words and Phrases: Chaotic dynamical system, Invariant measure, Correlation dimension, U-statistics.

\section{Introduction}

We consider trajectory $\left\{X_{t}\right\}_{t=1,2, \ldots, N}$ generated by dynamical system

$$
X_{t}=F\left(X_{t-1}, X_{t-2}, \ldots, X_{t-d}\right),
$$

where $F: \mathbf{R}^{d} \rightarrow \mathbf{R}$ is unknown nonlinear function, $d$ is unknown positive integer called embedding dimension. This system may be represented as

$$
\mathbf{Y}_{t}=\mathbf{F}\left(\mathbf{Y}_{t-1}\right)
$$

where $\mathbf{F}(\mathbf{x})={ }^{t}\left(F(\mathbf{x}), x_{1}, \ldots, x_{d-1}\right)$ for $\mathbf{x}={ }^{t}\left(x_{1}, x_{2}, \ldots, x_{d}\right)$ and $\mathbf{Y}_{t}={ }^{t}\left(X_{t}, X_{t-1}\right.$, $\left.\ldots, X_{t-d+1}\right)$. Assuming $\mathbf{Y}_{t} \in \Omega$ for closed $\Omega \subseteq \mathbf{R}^{d}$ and also assuming the ergodicity of $\left\{\mathbf{Y}_{t}\right\}$, we formulate the system as $(\Omega, \mathcal{F}, \mu, \mathbf{F})$, where $\mathcal{F}$ is the completion of the Borel $\sigma$-field with respect to $\mu$, and $\mu$ is an invariant measure, i.e. $\mu\left(\mathrm{F}^{-1} A\right)=\mu(A)$ for $A \in \mathcal{F}$.

Putting

$$
C(r)=\iint_{\Omega \times \Omega} I\left(\left\|\mathbf{y}_{1}-\mathbf{y}_{2}\right\| \leq r\right) d \mu\left(\mathbf{y}_{1}\right) d \mu\left(\mathbf{y}_{2}\right),
$$

where $I$ denotes an indicator function and $\|\cdot\|$ is a norm, Grassberger and Procaccia $(1983 a, b)$ defined the correlation dimension as

$$
\nu=\lim _{r \rightarrow 0} \frac{\log C(r)}{\log r}
$$

if the limit exists. The correlation dimension was introduced as a measure for representing the fractal dimension of the attractor of $\left\{\mathbf{Y}_{t}\right\}$. Estimating the dimension of an attractor of chaotic dynamical systems can provide useful, even vital information for understanding the dynamical systems (see for example, Abraham et al. 1989).

\footnotetext{
* Graduate School of Mathematics, Kyushu University Hakozaki 6-10-1 Higashi-Ku Fukuoka 812-8581 Japan. Email: atsushi@math.kyushu-u.ac.jp
} 
If $\mu$ is finite, it may be normalized to total mass 1 and may be interpreted as the limiting probability distribution of $\left\{\mathbf{Y}_{t}\right\}$. Thus, it is possible to formalize concepts such as the consistency of an estimator even though it is deterministic. The references on randomness of dynamical systems include Denker and Keller (1986), Jensen (1993), and Serinko (1994).

Our goal of this paper is to show the consistency of the estimator of the correlation dimension proposed by Kawaguchi (2002) which is represented in Section 2.

Serinko (1994) provided a consistent estimator of the correlation dimension as follows. Putting

$$
C_{N}(r, \mathbf{Y})=\left(\begin{array}{c}
N \\
2
\end{array}\right)^{-1} \sum_{i<j}^{N} I\left(\left\|\mathbf{Y}_{i}-\mathbf{Y}_{j}\right\| \leq r\right)
$$

and $r_{j}^{\left(m_{N}\right)}=r_{0}^{2 m_{N}+1-j}, j=1,2, \ldots,\left[m_{N}\right]$ where some $0<r_{0}<1$ and $m_{N}=$ $\frac{1}{2}\left(1+\frac{\log r_{N}}{\log s}\right)$ for any $0<s<1$ and $\left\{r_{N}\right\}$ that tends to 0 as $N \rightarrow \infty$, the estimator is given by

$$
\hat{\nu}_{N}^{(S)}=\sum_{j=1}^{\left[m_{N}\right]}\left(u_{j}-\bar{u}\right) \log C_{N}\left(r_{j}^{\left(m_{N}\right)}, \mathbf{Y}\right) / \sum_{j=1}^{\left[m_{N}\right]}\left(u_{j}-\bar{u}\right)^{2},
$$

where $u_{j}=\log r_{j}^{\left(M_{N}\right)}$ and $\bar{u}=\left[m_{N}\right]^{-1} \sum_{j=1}^{\left[m_{N}\right]} u_{j}$.

Serinko (1994) showed, under some conditions on dynamical system, which include the uniform mixing condition known as the weak Bernoulli property, that there exists a sequence of reals $\left\{b_{N}\right\}_{N=1}^{\infty}$ (see below) that tends to 0 as $N$ goes to infinity, such that for any other sequence of real $\left\{r_{N}\right\}_{N=1}^{\infty}$ that tends to 0 no faster than $\left\{b_{N}\right\}$, and $\varepsilon>0$, then

$$
\lim _{N \rightarrow \infty} \mu\left(\left|\hat{\nu}_{N}^{(S)}-\nu\right|>\varepsilon\right)=0
$$

Note that Serinko (1994) also gave $r_{N}$ as $r_{N}=N^{-\frac{1}{2\left(d+\varepsilon_{0}\right)}}$ for some $\varepsilon_{0}>0$ in the corollary. From a practical point of view, Serinko's estimator does not have an obvious advantage over the usual least-squares estimator, since the $m_{N}$ depend on unknown parameter $\varepsilon_{0}$, and has no clear practical value. Our estimator has the same form as Serinko's estimator except for $m_{N}$. Thus, we take the same approach as Serinko (1994) for the proof of the consistency.

This paper is organized as follows. We introduce the estimator and give the theorem in Section 2. In Section 3, the consistency of the estimator is proved.

\section{The estimator and theorem} Let

We recall the estimator of correlation dimension proposed by Kawaguchi (2002).

$$
C_{N 2}(r, \mathbf{Y})=\left(\begin{array}{c}
N \\
3
\end{array}\right)^{-1} \sum_{i \neq j, i \neq k, j \neq k}^{N} I\left(\left\|\mathbf{Y}_{i}-\mathbf{Y}_{j}\right\| \leq r,\left\|\mathbf{Y}_{i}-\mathbf{Y}_{k}\right\| \leq r\right)
$$


For some given $0<s<1$, and $r_{0}>1$, putting $r_{j}^{\left(M_{N}\right)}=r_{0} s^{M_{N}-\left[\frac{M_{N}}{2}\right]+j},(j=$ $\left.0,1, \ldots, L_{N}=\left[\frac{M_{N}}{2}\right]\right)$ where

$$
M_{N}=\max \left\{m \in \mathbf{Z}_{>0} ; C_{N 2}\left(r_{m}, \mathbf{Y}_{N}\right) \neq 0, \text { for } r_{m}=r_{0} s^{m}\right\},
$$

the estimator of $\nu$ is given by

$$
\hat{\nu}_{N}=\sum_{j=0}^{L_{N}}\left(u_{j}-\bar{u}\right) \log C_{N}\left(r_{j}^{\left(M_{N}\right)}, \mathbf{Y}\right) / \sum_{j=0}^{L_{N}}\left(u_{j}-\bar{u}\right)^{2}
$$

where $u_{j}=\log r_{j}^{\left(M_{N}\right)}$ and $\bar{u}=\left(L_{N}+1\right)^{-1} \sum_{j=0}^{L_{N}} u_{j}$.

The consistency of this estimator is given in next theorem.

THEOREM 2.1. Under the assumptions that are given in the following section, it follows that for any $\varepsilon>0$,

$$
\lim _{N \rightarrow \infty} \mu\left(\left|\hat{\nu}_{N}-\nu\right|>\varepsilon\right)=0
$$

\section{Proof of theorem 2.1}

At first, we decompose the estimator as follows.

LemMa 3.1. (Serinko, 1994)

$$
\hat{\nu}_{N}=\nu+d_{N}+e_{N}
$$

where

$$
\begin{aligned}
& d_{N}= \frac{1}{S_{u u}} \sum_{j=0}^{L_{N}}\left(\log C\left(r_{j}^{\left(M_{N}\right)}\right)-\nu \log r_{j}^{\left(M_{N}\right)}\right)\left(u_{j}-\bar{u}\right) \\
& e_{N}= \frac{1}{S_{u u}} \sum_{j=0}^{L_{N}}\left(\log C_{N}\left(r_{j}^{\left(M_{N}\right)}, \mathbf{Y}\right)-\log C\left(r_{j}^{\left(M_{N}\right)}\right)\right)\left(u_{j}-\bar{u}\right), \text { and } \\
& S_{u u}=\sum_{j=0}^{L_{N}}\left(u_{j}-\bar{u}\right)^{2} .
\end{aligned}
$$

Next, we prove that $d_{N}$ tends to 0 as $N \rightarrow \infty$ in probability. We assume the following assumption.

Assumption 3.2. For any $r>0$ and $\varepsilon>0$,

$$
\lim _{N \rightarrow \infty} \mu\left(C_{N 2}(r, \mathbf{Y})>\varepsilon\right)=1 .
$$

Lemma 3.3. Under Assumption 3.2, for $M_{N}$ in (2.1),

$$
\mu\left(\lim _{N \rightarrow \infty} M_{N}=\infty\right)=1
$$


Proof. For any integers $m$ and $N_{0}$, let

$$
A\left(m, N_{0}\right)=\left\{\omega \in \Omega ; C_{N 2}\left(r_{l}, \mathrm{Y}\right)>\varepsilon\right.
$$

$$
\text { for all } \left.N \geq N_{0}, l=0,1, \ldots, m \text {, and } \varepsilon>0 \text { where } r_{l}=r_{0} s^{l}\right\} \text {. }
$$

Then, $A\left(m, N_{0}\right) \subset\left\{M_{N} \geq m\right.$ for $\left.N \geq N_{0}\right\}$, and therefore

$$
\begin{aligned}
\mu\left(\lim _{N \rightarrow \infty} M_{N} \geq m\right) & \geq \mu\left(\bigcap_{N_{0}=1}^{\infty} A\left(m, N_{0}\right)\right) \\
& =\lim _{N_{0} \rightarrow \infty} \mu\left(A\left(m, N_{0}\right)\right) .
\end{aligned}
$$

From Assumption 3.2, the proof is completed.

Lemma 3.4. Under Assumption 3.2, for any $\varepsilon>0$,

$$
\lim _{N \rightarrow \infty} \mu\left(\left|d_{N}\right|>\varepsilon\right)=0
$$

Proof. First, from the definition of the correlation dimension,

$$
\frac{\log C(r)-\nu \log r}{\log r} \rightarrow 0, \quad(r \rightarrow 0) .
$$

Thus, there exists a positive real $A(r) \rightarrow 0$ as $r \rightarrow 0$ such that for sufficiently small $r>0$,

$$
|\log C(r)-\nu \log r| \leq A(r)|\log r|
$$

Next, it follows by the Cauchy-Schwarz inequality that

$$
\begin{aligned}
\left|d_{N}\right| & \leq \frac{1}{S_{u u}} \sqrt{\sum_{j=0}^{L_{N}}\left\{\log C\left(r_{j}^{\left(M_{N}\right)}\right)-\nu \log r_{j}^{\left(M_{N}\right)}\right\}^{2}} \sqrt{\sum_{j=0}^{L_{N}}\left(u_{j}-\bar{u}\right)^{2}} \\
& \leq \frac{1}{\sqrt{S_{u u}}} \sqrt{\sum_{j=0}^{L_{N}}\left\{A\left(r_{j}^{\left(M_{N}\right)}\right) \log r_{j}^{\left(M_{N}\right)}\right\}^{2}} \\
& \leq \frac{1}{\sqrt{S_{u u}}} \times \max _{0 \leq j \leq L_{N}} A\left(r_{j}^{\left(M_{N}\right)}\right) \times \sqrt{\sum_{j=0}^{L_{N}}\left\{\log r_{j}^{\left(M_{N}\right)}\right\}^{2}} .
\end{aligned}
$$

Serinko(1994) gave

$$
S_{u u}=\frac{1}{12} L_{N}\left(L_{N}+1\right)\left(L_{N}+2\right)(\log s)^{2} .
$$

Thus, from Lemma 3.3 and the definition of $L_{N}$,

$$
\sqrt{S_{u u}}=O_{p}\left(M_{N}^{\frac{3}{2}}\right) \text {. }
$$

On the other hand, from Lemma 3.3

$$
\sqrt{\sum_{j=0}^{L_{N}}\left\{\log r_{j}^{\left(M_{N}\right)}\right\}^{2}}=O_{p}\left(M_{N}^{\frac{3}{2}}\right)
$$


and

$$
\mu\left(\lim _{N \rightarrow \infty} A\left(r_{j}^{\left(M_{N}\right)}\right)=0\right)=1
$$

Therefore,

$$
\mu\left(\lim _{N \rightarrow \infty}\left\{\frac{1}{\sqrt{S_{u u}}} \times \max _{0 \leq j \leq L_{N}} A\left(r_{j}^{\left(M_{N}\right)}\right) \times \sqrt{\sum_{j=0}^{L_{N}}\left\{\log r_{j}^{\left(M_{N}\right)}\right\}^{2}}\right\}=0\right)=1
$$

and

$$
\mu\left(\lim _{N \rightarrow \infty}\left|d_{N}\right|=0\right)=1 .
$$

The convergence with probability 1 implies the convergence in probability, therefore the proof is completed.

Next, we prove that $e_{N}$ tends to 0 as $N \rightarrow \infty$ in probability. In order to state the next theorem, we give some notations and assumptions. Let $\alpha=\left\{A_{1}, A_{2}, \ldots, A_{m}\right\}$ and $\beta=\left\{B_{1}, B_{2}, \ldots, B_{n}\right\}$ be finite measurable partitions of $\Omega$. From these one may construct the following partitions:

1. $\alpha \vee \beta=\{A \cap B ; A \in \alpha, B \in \beta\}$

2. $\mathbf{F}^{-1} \alpha=\left\{\mathbf{F}^{-1} A ; A \in \alpha\right\}$

3. $\alpha_{r}^{s}=\mathbf{F}^{(-r)} \alpha \vee \mathbf{F}^{(-r-1)} \alpha \vee \cdots \vee \mathbf{F}^{(-s+1)} \alpha \vee \mathbf{F}^{(-s)} \alpha \quad(r, s \in \mathbf{N}$, s.t. $r<s)$, where $\mathbf{F}^{(k)}$ is $k$ times convolution of $\mathbf{F}$.

Let $\mathcal{F}_{r}^{s}$ denote the $\sigma$-algebra generated by $\alpha_{r}^{s}(r, s \in \mathbf{N}$, s.t. $r<s)$, and $\mathcal{F}_{0}^{\infty}$ denote the smallest $\sigma$-algebra which contains all of the $\mathcal{F}_{r}^{s}(r, s \in \mathbf{N}$, s.t. $r<s)$.

\section{DEFINITION 3.5. (Generator)}

$$
\alpha \text { is generator } \Longleftrightarrow \mathcal{F}_{0}^{\infty}=\mathcal{F}
$$

Definition 3.6. (Weak Bernoulli)

A measurable partition $\alpha$ is said to be weak Bernoulli for dynamical system if

$$
\beta_{k}=\sup _{r, s \in \mathbb{N}} \sum_{A \in \alpha_{0}^{r}} \sum_{B \in \alpha_{r+k}^{r+s+k}}|\mu(A \cap B)-\mu(A) \mu(B)|
$$

goes to zero as $k \rightarrow \infty$. The $\beta_{k}$ 's are called the mixing coefficients.

We assume the following assumptions for the dynamical system $(\Omega, \mathcal{F}, \mu, \mathbf{F})$.

Assumption 3.7. $(\Omega, \mathcal{F}, \mu, \mathbf{F})$ has the measurable partition $\alpha$ which is weak Bernoulli and generator.

AsSUmption 3.8. $(\Omega, \mathcal{F}, \mu, \mathbf{F})$ is such that the mixing coefficients satisfy

$$
\beta_{k}^{\frac{\delta}{2+\delta}}=O\left(k^{-(1+\varepsilon)}\right)
$$

for some $\delta>0$ and $0<\varepsilon<1$. 
Let $\eta^{(l)}(r)=\left\|\mu\left(\bar{B}_{r}\left(\mathbf{Y}_{j}\right)\right)-\mu\left(\bar{B}_{r}\left(\mathbf{Y}_{j}^{(l)}\right)\right)\right\|_{2}^{2}$, where $\bar{B}_{r}(\mathbf{y})=\{\mathbf{x} ;\|\mathbf{x}-\mathbf{y}\| \leq r\}$ and $\mathbf{Y}_{j}^{(l)}=E\left[\mathbf{Y}_{j} \mid \alpha_{j}^{j+l}\right], j=1,2, \cdots$ Let $\bar{\eta}^{(l)}=\sup _{r} \eta^{(l)}(r)$.

Assumption 3.9. $(\Omega, \mathcal{F}, \mu, \mathbf{F})$ is such that

$$
\bar{\eta}^{(l) \frac{1}{2}}=o\left(l^{-(1+\gamma)}\right)
$$

for some $\gamma>0$ and $\gamma /(1+\gamma)>\varepsilon$.

Let $\psi_{k}^{(l)}(r)=\left\|I\left(\left\|\mathbf{Y}_{i}-\mathbf{Y}_{j}\right\| \leq r\right)-I\left(\left\|\mathbf{Y}_{i}^{(l)}-\mathbf{Y}_{j}^{(l)}\right\| \leq r\right)\right\|_{2}^{2}$ for $i, j=1,2, \cdots$ and $k=|i-j|$, and $\bar{\psi}_{k}^{(l)}=\sup _{r} \psi_{k}^{(l)}(r)$.

ASSUMPTION 3.10. $(\Omega, \mathcal{F}, \mu, \mathbf{F})$ is such that for any sequence of reals $\left\{c_{n}\right\}_{n=0}^{\infty}$ satisfying $\lim _{n \rightarrow \infty} c_{n}=\infty$ and $c_{n}=o\left(n^{\frac{1}{2}}\right)$, one has

$$
\sum_{k=0}^{n-1} \bar{\psi}_{k}^{\left(c_{n}\right) \frac{1}{2}}=o\left(n^{\frac{1}{2}}\right) \text {. }
$$

Let $b_{N}=\left(\frac{1}{N}\right)^{\frac{1}{2\left(\nu+\varepsilon_{0}\right)}}$, where some $\varepsilon_{0}>0$.

THEOREM 3.11. (Serinko, 1994)

If $(\Omega, \mathcal{F}, \mu, \mathbf{F})$ satisfies Assumption 3.7 through 3.10, then whenever $\nu$ exists,

$$
\lim _{N \rightarrow \infty} r_{N}=0 \quad \text { and } \quad \limsup _{N \rightarrow \infty} \frac{b_{N}}{r_{N}}<\infty
$$

imply

$$
\lim _{N \rightarrow \infty} \mu\left(\left|\frac{C_{N}\left(r_{N}, \mathbf{Y}\right)-C\left(r_{N}\right)}{C\left(r_{N}\right)}\right|>\varepsilon\right)=0 .
$$

Assumption 3.12. For some $\varepsilon_{0}>0$ and some $\delta<\frac{1}{d+\varepsilon_{0}}$, there exists $N_{0} \in \mathbf{N}$ such that for any $N>N_{0}$,

$$
M_{N}>\frac{\delta \log N}{2|\log s|} \quad \text { a.e.. }
$$

LEMMA 3.13. Under Assumption 3.12, let $r_{N}=r_{0} s^{M_{N}}$, then

$$
\lim _{N \rightarrow \infty} r_{N}=0 \quad \text { and } \quad \limsup _{N \rightarrow \infty} \frac{b_{N}}{r_{N}}<\infty \quad \text { a.e.. }
$$

PROOF. For the former part of lemma, we obtain immediately from Lemma 3.3. Setting $r^{\prime}{ }_{N}=N^{\frac{-1}{2\left(d+\varepsilon_{0}\right)}}, \operatorname{Serinko(1994)}$ proved

$$
\limsup _{N \rightarrow \infty} \frac{b_{N}}{r^{\prime} N}<\infty
$$

Thus, the last part of lemma is proved if we prove $r_{N}>r^{\prime}{ }_{N}$ a.e. for any $N>N_{0}$. From Assumption 3.12, we have

$$
\begin{aligned}
\log \frac{r_{N}}{r^{\prime} N} & =\log \frac{r_{0} s^{M_{N}}}{N^{\frac{-1}{2\left(d+\varepsilon_{0}\right)}}} \\
& =\log r_{0}+M_{N} \log s+\frac{1}{2\left(d+\varepsilon_{0}\right)} \log N \\
& >\log r_{0}+\left(-\delta+\frac{1}{d+\varepsilon_{0}}\right) \frac{1}{2} \log N>0 .
\end{aligned}
$$


Therefore, the proof is completed.

LEMMA 3.14. Under the condition of Theorem 3.11 and Assumption 3.12, for any $\varepsilon>0$,

$$
\lim _{N \rightarrow \infty} \mu\left(\left|e_{N}\right|>\varepsilon\right)=0 .
$$

Proof. It follows by the Cauchy-Schwarz inequality that

$$
\begin{aligned}
\left|e_{N}\right| \leq & \frac{1}{S_{u u}} \sqrt{\sum_{j=0}^{L_{N}}\left\{\log C_{N}\left(r_{j}^{\left(M_{N}\right)}, \mathbf{Y}\right)-\log C\left(r_{j}^{\left(M_{N}\right)}\right)\right\}^{2}} \sqrt{\sum_{j=0}^{L_{N}}\left(u_{j}-\bar{u}\right)^{2}} \\
\leq & \frac{1}{\sqrt{S_{u u}}} \sqrt{L_{N}+1} \max _{0 \leq j \leq L_{N}}\left|\log C_{N}\left(r_{j}^{\left(M_{N}\right)}, \mathbf{Y}\right)-\log C\left(r_{j}^{\left(M_{N}\right)}\right)\right| \\
= & \left\{\frac{1}{12}(\log s)^{2} L_{N}\left(L_{N}+2\right)\right\}^{-\frac{1}{2}} \\
& \times \max _{0 \leq j \leq L_{N}}\left|\log C_{N}\left(r_{j}^{\left(M_{N}\right)}, \mathbf{Y}\right)-\log C\left(r_{j}^{\left(M_{N}\right)}\right)\right|
\end{aligned}
$$

Moreover,

$$
\begin{array}{r}
\mu\left(\left\{\frac{1}{12}(\log s)^{2} L_{N}\left(L_{N}+2\right)\right\}^{-\frac{1}{2}} \times \max _{0 \leq j \leq L_{N}}\left|\log C_{N}\left(r_{j}^{\left(M_{N}\right)}, \mathbf{Y}\right)-\log C\left(r_{j}^{\left(M_{N}\right)}\right)\right|>\varepsilon\right) \\
\leq \mu\left(\left\{\frac{1}{12}(\log s)^{2} L_{N}\left(L_{N}+2\right)\right\}^{-\frac{1}{2}}>1\right) \\
+\mu\left(\max _{0 \leq j \leq L_{N}}\left|\log C_{N}\left(r_{j}^{\left(M_{N}\right)}, \mathbf{Y}\right)-\log C\left(r_{j}^{\left(M_{N}\right)}\right)\right|>\varepsilon\right) .
\end{array}
$$

From Lemma 3.3,

$$
\lim _{N \rightarrow \infty} \mu\left(\left\{\frac{1}{12}(\log s)^{2} L_{N}\left(L_{N}+2\right)\right\}^{-\frac{1}{2}}>1\right)=0 .
$$

For $r_{N}$ satisfied condition of Theorem 3.11,

$$
\begin{aligned}
\mu & \left(\left|\log C_{N}\left(r_{N}, \mathbf{Y}\right)-\log C\left(r_{N}\right)\right|>\varepsilon\right)=\mu\left(\left|\log \frac{C_{N}\left(r_{N}, \mathbf{Y}\right)}{C\left(r_{N}\right)}\right|>\varepsilon\right) \\
& =\mu\left(\log \frac{C_{N}\left(r_{N}, \mathbf{Y}\right)}{C\left(r_{N}\right)}>\varepsilon\right)+\mu\left(\log \frac{C_{N}\left(r_{N}, \mathbf{Y}\right)}{C\left(r_{N}\right)}<-\varepsilon\right) \\
& =\mu\left(\frac{C_{N}\left(r_{N}, \mathbf{Y}\right)}{C\left(r_{N}\right)}>e^{\varepsilon}\right)+\mu\left(\frac{C_{N}\left(r_{N}, \mathbf{Y}\right)}{C\left(r_{N}\right)}<e^{-\varepsilon}\right) \\
& =\mu\left(\frac{C_{N}\left(r_{N}, \mathbf{Y}\right)-C\left(r_{N}\right)}{C\left(r_{N}\right)}>e^{\varepsilon}-1\right)+\mu\left(\frac{-C_{N}\left(r_{N}, \mathbf{Y}\right)+C\left(r_{N}\right)}{C\left(r_{N}\right)}>1-e^{-\varepsilon}\right) .
\end{aligned}
$$

This tends to 0 as $N \rightarrow \infty$ from Theorem 3.11. Therefore, from Lemma 3.13,

$$
\lim _{N \rightarrow \infty} \mu\left(\max _{0 \leq j \leq L_{N}}\left|\log C_{N}\left(r_{j}^{\left(M_{N}\right)}, \mathbf{Y}\right)-\log C\left(r_{j}^{\left(M_{N}\right)}\right)\right|>\varepsilon\right)=0 .
$$

Hence, the proof is completed. 
ProOF OF THEOREM 2.1.

From Lemma 3.1, for any $\varepsilon>0$,

$$
\mu\left(\left|\hat{\nu}_{N}-\nu\right|>\varepsilon\right) \leq \mu\left(\left|d_{N}\right|>\varepsilon / 2\right)+\mu\left(\left|e_{N}\right|>\varepsilon / 2\right) .
$$

Hence from Lemma 3.4 and Lemma 3.14,

$$
\lim _{N \rightarrow \infty} \mu\left(\left|\hat{\nu}_{N}-\nu\right|>\varepsilon\right)=0 .
$$

The proof of Theorem 2.1 is completed.

\section{Acknowledgement}

Author would express sincere gratitude to Prof. Takashi Yanagawa for helpful comment and suggestions. Also author would like to deep thanks to Dr. Koji Yonemoto for his valuable advice. This work is supported by Kyushu University 21st Century COE Program, Development of Dynamic Mathematics with High Functionality, of the Ministry of Education, Culture, Sports, Science and Technology of Japan.

\section{References}

Abraham, N. B. Albano, A. M. Passamante, A. and Rapp, P. E. (1989). Measure of Complexity and Chaos. New York: Plenum.

Denker, M. and Keller, G. (1986). Rigourous Statistical Procedures for Data from Dynamic Systems, Journal of Statistical Physics, 44, No.1-2, 67-93.

Grassberger, P. and Procaccia, I. (1983a). Characterization for Strange Attractors, Physical Review Letters, 50, No.5, 346-349.

Grassberger, P. and Procaccia, I. (1983b). Measuring the strangeness of strange attractors, Physica D, 9, No.5, 189-208.

Jensen, J. L. (1993). Chaotic dynamical systems with a view toward statistics: A review, in Networks and Chaots-Statistical and Probabilistic Aspects, Barndorff-Nielsen, O. E., Jensen, J. L., and Kendall, W. S., Eds. London, U. K.: Chapman and Hall.

Kawaguchi, A. (2002). Estimating the correlation dimension from chaotic dynamical systems by U-statistics, Bulletin of Informatics and Cybernetics, 34, No.2, 143-150.

Serinko, R. J. (1994). Consistent Approach to Least Squares Estimation of Correlation Dimension in Weak Bernoulli Dynamical Systems, The annals of Applied Probability, 4, No.4, 1234-1254.

Received February 6, 2004

Revised March 10, 2004 